\title{
Next Generation Nuclear Plant Resilient Control System Functional Analysis
}

The INL is a

U.S. Department of Energy National Laboratory

operated by

Battelle Energy Alliance

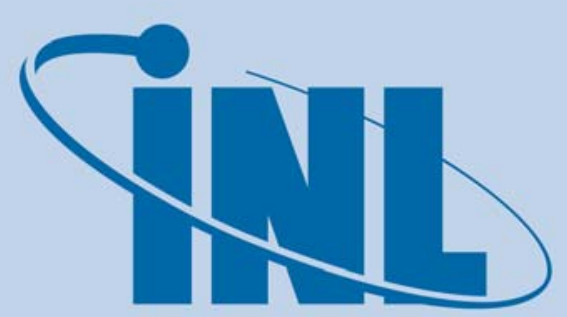

Idaho National Laboratory
Lynne M. Stevens

July 2010 


\section{DISCLAIMER}

This information was prepared as an account of work sponsored by an agency of the U.S. Government. Neither the U.S. Government nor any agency thereof, nor any of their employees, makes any warranty, expressed or implied, or assumes any legal liability or responsibility for the accuracy, completeness, or usefulness, of any information, apparatus, product, or process disclosed, or represents that its use would not infringe privately owned rights. References herein to any specific commercial product, process, or service by trade name, trade mark, manufacturer, or otherwise, does not necessarily constitute or imply its endorsement, recommendation, or favoring by the U.S. Government or any agency thereof. The views and opinions of authors expressed herein do not necessarily state or reflect those of the U.S. Government or any agency thereof. 


\title{
Next Generation Nuclear Plant Resilient Control System Functional Analysis
}

\author{
Lynne M. Stevens
}

July 2010

\begin{abstract}
Idaho National Laboratory
Next Generation Nuclear Plant Project

Idaho Falls, Idaho 83415
\end{abstract}

Prepared for the

U.S. Department of Energy

Office of Nuclear Energy

Under DOE Idaho Operations Office

Contract DE-AC07-05ID14517 

Next Generation Nuclear Plant Project

Next Generation Nuclear Plant Resilient Control System Functional Analysis

INL/EXT-10-19359

July 2010
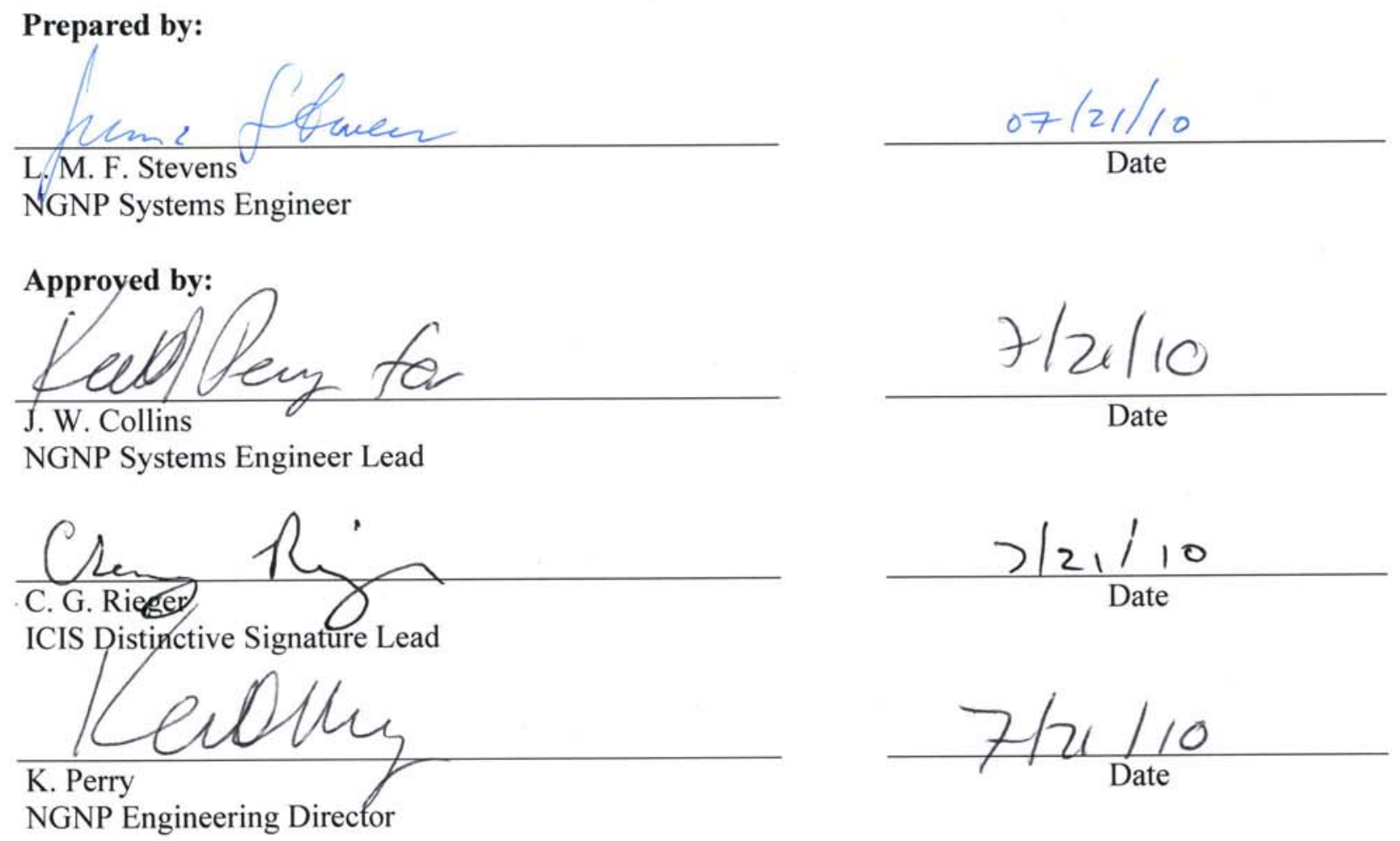



\begin{abstract}
Control Systems and their associated instrumentation must meet reliability, availability, maintainability, and resiliency criteria in order for high temperature gas-cooled reactors (HTGRs) to be economically competitive. Research, perhaps requiring several years, may be needed to develop control systems to support plant availability and resiliency. This report functionally analyzes the gaps between traditional and resilient control systems as applicable to HTGRs, which includes the Next Generation Nuclear Plant; defines resilient controls; assesses the current state of both traditional and resilient control systems; and documents the functional gaps existing between these two controls approaches as applicable to HTGRs. This report supports the development of an overall strategy for applying resilient controls to HTGRs by showing that control systems with adequate levels of resilience perform at higher levels, respond more quickly to disturbances, increase operational efficiency, and increase public protection.
\end{abstract}




\section{SUMMARY}

A preeminent objective for corporate and government organizations is the protection of major investments, which is attained by achieving state awareness, a comprehensive understanding of security and safety, for critical infrastructures. ${ }^{1}$ Given the dependence of critical infrastructure on control systems for automation, the integrity of these systems and their ability to provide owner/operators a high degree of state awareness is essential in attaining a high degree of investment protection and public acceptance. Operators as well as government are therefore burdened to ensure they have a timely understanding of the status of their plant or all plants, respectively, to ensure efficient operations and investment and public protection. "This characterization is a significant objective that must consider many aspects of instrumentation, control, and intelligent systems in order to achieve the required result. These aspects include sensory, communication, analysis, decision, and human system interfaces necessary to achieve fusion of data and presentation of results that will provide an understanding of what issues are important and why."

A resilient system can be defined as "one that maintains state awareness and an accepted level of operational normalcy in response to disturbances, including threats of an unexpected and malicious nature." The framework for a resilient control system contains principles and methods that proactively recognize threats, integrate automation and human response, provide robust and adaptive mechanisms, consider all threats and events, and tailor information to the consumer. These methods and principles improve a system's ability to anticipate, perceive, respond and adapt. Improvements to these four abilities increase the system's overall ability to perform its necessary functions and operate resiliently. As this paper will show, control systems with adequate levels of resilience perform at higher levels and respond more quickly to disturbances.

The key features of a resilient control system are state awareness and resilient design. State awareness is the top down view, where understanding exists of all aspects that may affect performance, including stability, efficiency, physical security, and cyber security. Resilient design is a bottom up view, where the inherent design of the system is built to account for the areas of resilience. These areas of resilience are human systems, cyber awareness and complex, networked control systems. A notional measure of resilience is in the adaptive capacity, or the ability to respond to a threat and maintain acceptable functionality.

Unlike traditional control systems, resilient control systems of the future will be designed, installed, operated and maintained to survive a natural disaster, human error or intentional cyber attack with no loss of critical function. This is no small challenge in a sector that is complex, highly networked, and sensitive to the mildest failure. To achieve resilience and address the threats in next generation control systems, research is required to integrate an understanding of cyber security, human interaction and complex network design. The integration of these three aspects will be introduced in the concepts of data fusion, mixed initiative, and hierarchical control system design.

This report has been prepared for the Next Generation Nuclear Plant Project to functionally analyze the current state of traditional control systems, the current state of resilient control systems, and the gaps existing between traditional and resilient control systems as applicable to high temperature gas-cooled reactors (HTGRs). This report supports the development of an overall strategy for applying resilient controls to HTGRs by showing control systems with adequate levels of resilience perform at higher levels that respond more quickly to disturbances, provide more efficient operations, and increase public protection. 


\section{CONTENTS}

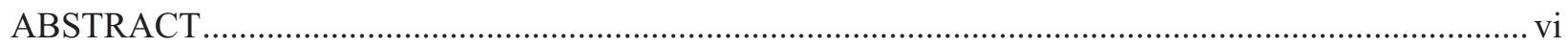

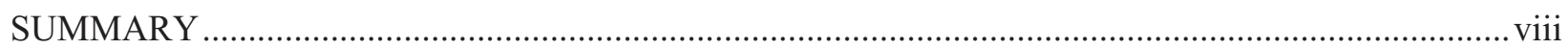

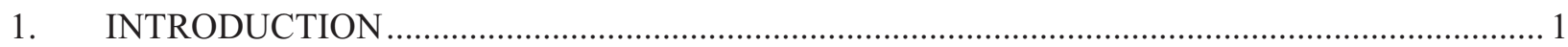

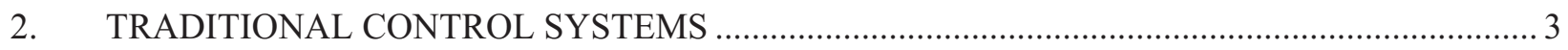

2.1 Traditional Control System Functional Framework \& Definitions........................................ 3

2.2 Traditional Control System Functional Flow ....................................................................... 4

2.3 Traditional Control System Operational Flow and Key Features ........................................ 4

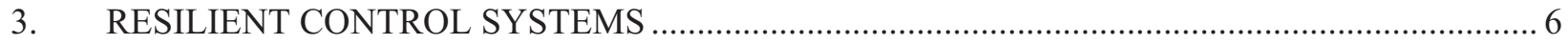

3.1 Resilient Control System Functional Framework and Definitions ..................................... 6

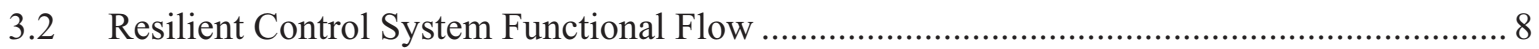

3.3 Resilient Control System Operational Flow and Key Features............................................ 10

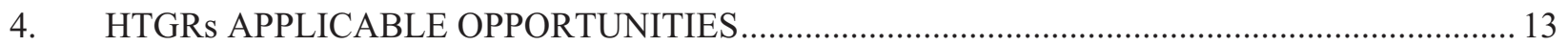

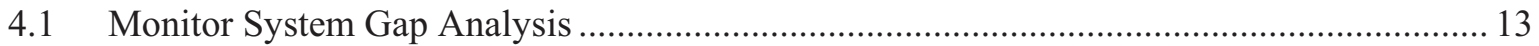

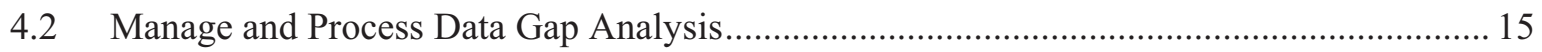

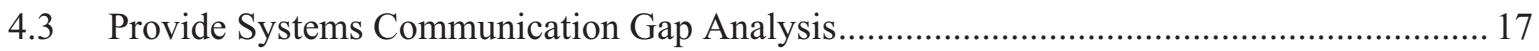

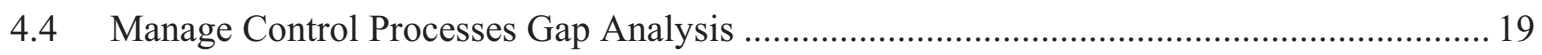

4.5 Benefits of Resilient Controls to HTGRs............................................................................. 21

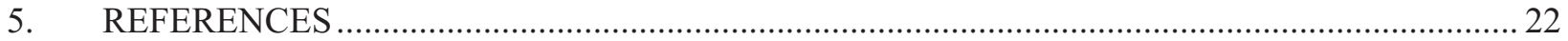

\section{FIGURES}

Figure 1. Control system performance level vs. time. …....................................................................

Figure 2. Traditional controls functional framework........................................................................ 3

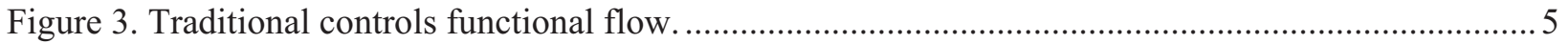

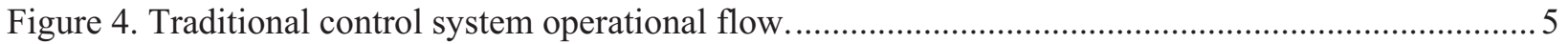

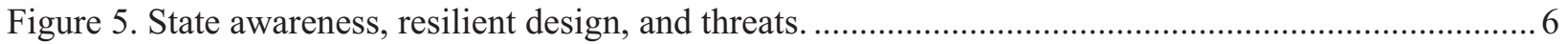

Figure 6. Additional functions performed by resilient controls............................................................ 7

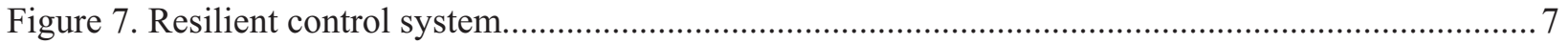

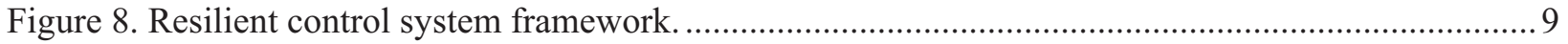

Figure 9. Functional flow additions of resilient control systems. .......................................................... 9

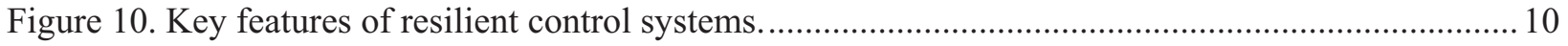

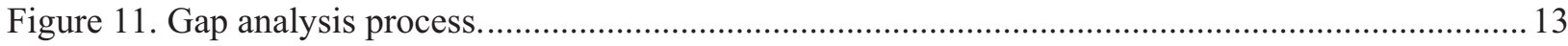

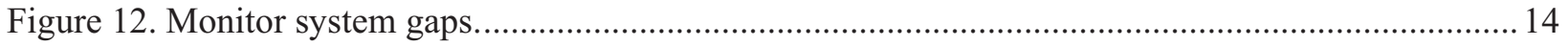




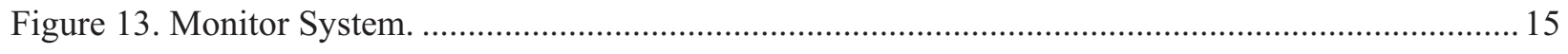

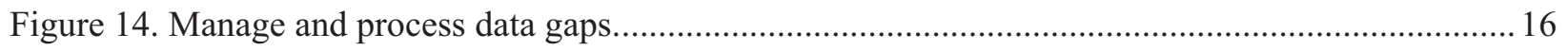

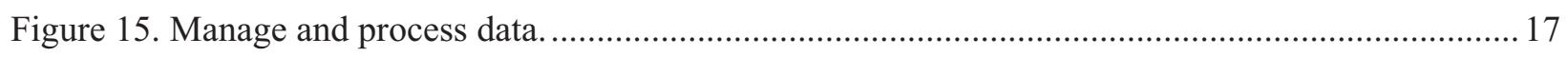

Figure 16. Provide Systems Communications Gaps..................................................................... 18

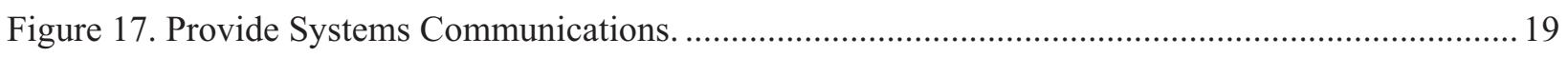

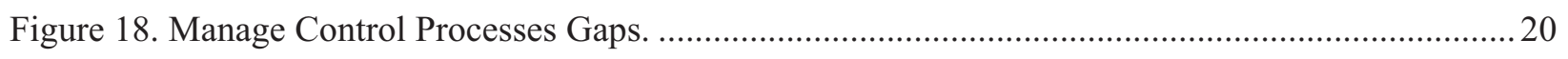

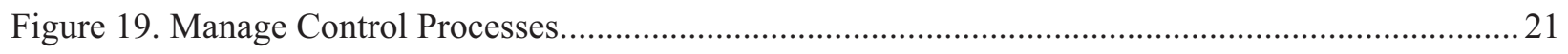




\section{Next Generation Nuclear Plant Resilient Control System Functional Analysis}

\section{INTRODUCTION}

Control Systems and their associated instrumentation must meet reliability, availability, maintainability, and resiliency criteria in order for high temperature gas-cooled reactors (HTGRs) to be economically competitive. Research, perhaps requiring several years, may be needed to develop control systems to support plant availability and resiliency. This report functionally analyzes the gaps between traditional and resilient control systems as applicable to HTGRs, which includes the Next Generation Nuclear Plant. This report defines resilient controls, assesses the current state of both traditional and resilient control systems, and documents the functional gaps existing between these two controls approaches as applicable to HTGRs. This report supports the development of an overall strategy for applying resilient controls to HTGRs by showing that control systems with adequate levels of resilience perform at higher levels, respond more quickly to disturbances, increase operational efficiency, and increase public protection.

A resilient system can be defined as "one that maintains state awareness and an accepted level of operational normalcy in response to disturbances, including threats of an unexpected and malicious nature." ${ }^{4}$ The key features of a resilient control system are state awareness and resilient design. State awareness is the top-down view, where understanding exists of all aspects that may affect performance, including stability, efficiency, physical security, and cyber security. Resilient design is a bottom-up view, where the inherent design of the system is built to account for the areas of resilience. These areas of resilience are human systems, cyber awareness, and complex networked control systems. A notional measure of resilience is in the adaptive capacity, or the ability to respond to a threat and maintain acceptable functionality.

An example of the need for resilient controls is found in the loss of Air France Flight AF447. The loss of reliable speed indication led to the disaster. A resilient control system might, in theory, determine the confidence in the reliability of pitot tube signals (maintaining state awareness) being used to control the airplane and replace them with other signals, for instance from a multiple global positioning system or other signals until the pitot tubes returned to being reliable. Research that might benefit the aircraft and other industries, such as the nuclear industry, could include identifying strategies for determining the confidence of a group of similar primary instruments and diverse secondary instruments and the methodology and other details in replacing the signals primary instrument with signals from the secondary instruments.

In the April 2010 edition of the Nuclear News, ${ }^{3}$ Congressman Markey asked the General Accounting Office to examine whether the Nuclear Regulatory Commission is seeking to evaluate and improve resilience to natural disasters (including earthquakes and storms) and to address potential climate-change issues such as cooling water availability and thermal discharge limits. To aid the Nuclear Regulatory Commission, Idaho National Laboratory is actively evaluating the improvements of resilience on HTGRs. Resilience is key in quickly identifying threats, providing essential operational information, and providing an adaptive capacity for response. As this strategy shows, control systems with adequate levels of resilience perform at higher levels and respond more quickly to disturbances.

Figure 1 shows that a resilient control system is more able to reduce the magnitude and duration of disruptive events. The effectiveness of a resilient system depends upon its ability to anticipate, absorb, adapt to, and rapidly recover from a potentially disruptive event. The increased performance over time provided by a resilient control system increases operational efficiency and public safety. 


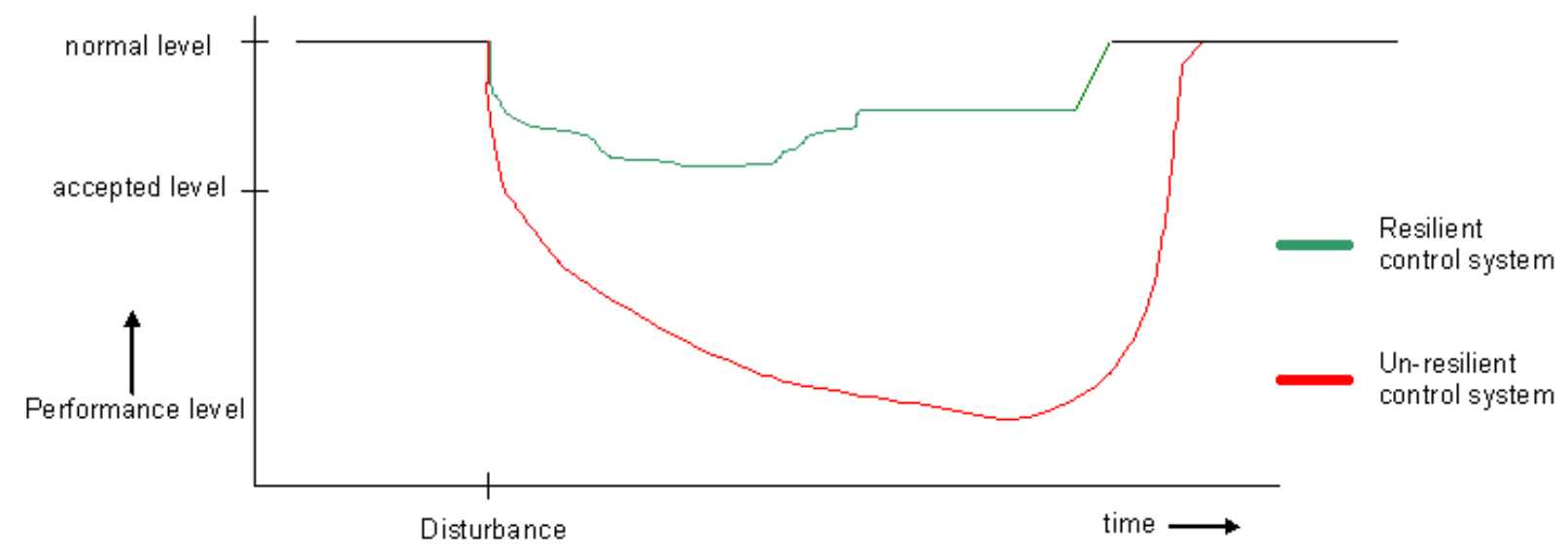

Figure 1. Control system performance level vs. time. 


\section{TRADITIONAL CONTROL SYSTEMS}

Since the advent of the proportional-integral-derivative feedback loop in the first half of last century, automatic control systems have developed from the original analog to now primarily digital technology. From the 1970s until now, many digital technologies have developed from programmable logic controllers to distributed control systems and supervisory control and data acquisition systems.

Control systems may be applied to a variety of industrial processes; be simple or complex; employ analog or digital components; and use manual control or significant automation with nonlinear controls, fuzzy logic, and process optimization. Regardless of application, most control systems perform the same basic functions in a similar flow. This section contains a high level description of the current traditional control systems commonly implemented in industrial process applications along with descriptions of common terminology.

\subsection{Traditional Control System Functional Framework \& Definitions}

A control system may be defined as "a device or set of devices to manage, command, direct or regulate the behavior of other devices or systems" record, regulate, supervise, authenticate, and (if necessary) restrict, the access to an asset, resource or system."

Figure 2 illustrates the functional framework diagram for a traditional control system.

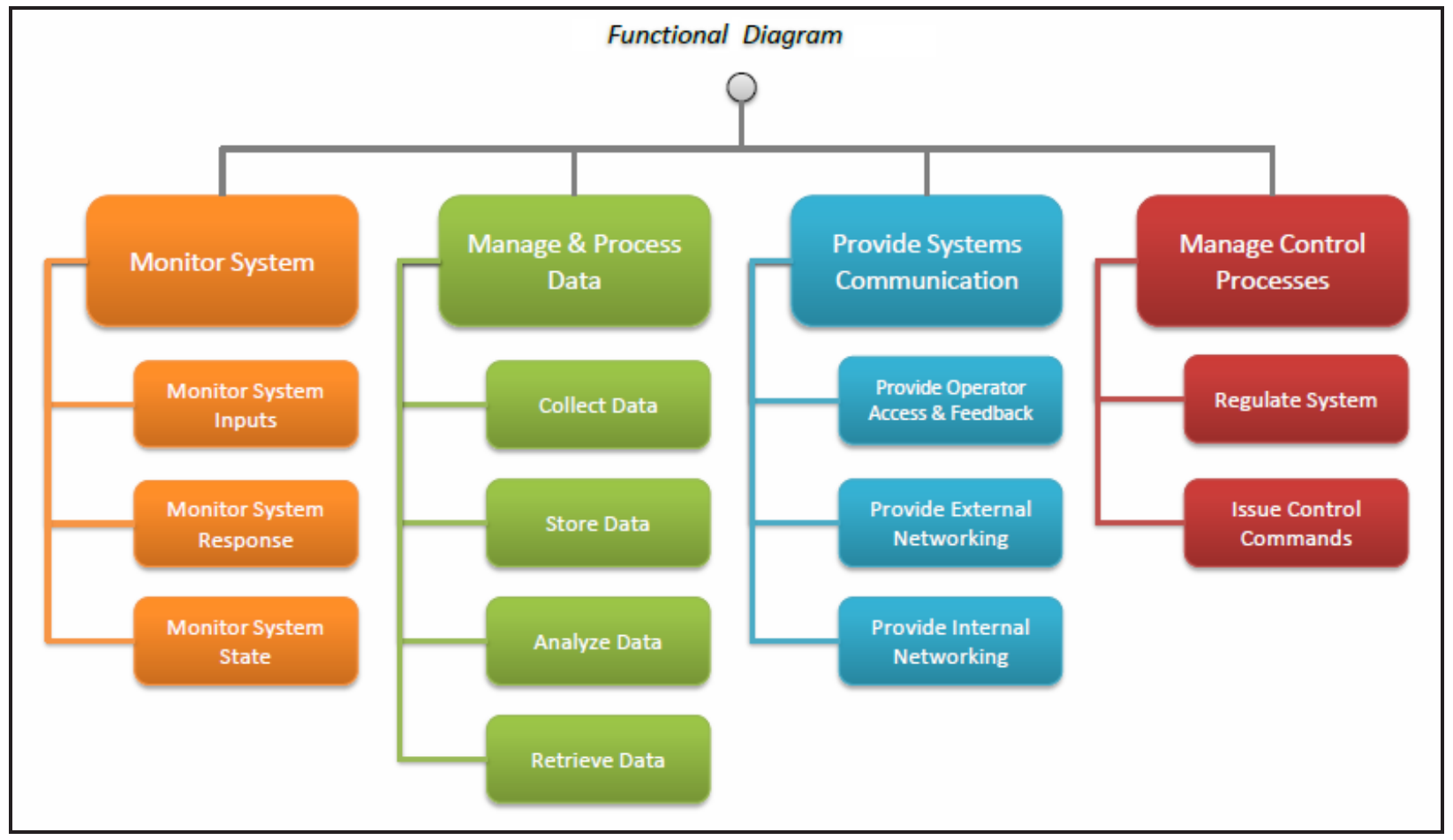

Figure 2. Traditional controls functional framework.

The Monitor System high-level function shown in Figure 2 contains the functions and processes for collecting state, input, and response data to measure a controlled system. The Monitor System Inputs function monitors instrument readings and observations about the controlled system. The monitor system response function monitors instrument readings and observations about outputs to the controlled system. 
The Monitor System State function characterizes the system and provides a picture of the system operability in the past or present.

The Manage and Process Data high-level function contains the functions and processes for directing the collection, processing, storage, analysis, and retrieval of system data. The Collect Data function receives raw analog or digital transmissions from instruments or observational sensors as data. The Store Data function refines, formats, or converts raw data suitable for subsequent secure storage available for immediate or future use. The Retrieve Data function obtains data from storage for analysis or system/operator use. The Analyze Data function performs engineering evaluation of data for purpose of highlighting useful information, suggesting conclusions, or supporting decision making.

The Provide Systems Communications high-level function contains the services, systems, and mechanisms that a control system uses to gather or provide information and/or the interaction of these services, systems or mechanisms with each other. The Provide Operator Access And Feedback function enables communications between the control system and a human. The Provide External Networking function enables communication between the control system and any external (to the control system) entity. The Provide Internal Networking function enables communication within the control system.

The Manage Control Processes high-level function shown in Figure 2 above contains the control functions for guiding, maintaining, or making changes to the entire controlled system by means of mechanical, optical, or electronic systems. The desired output is achieved by means of issuing control commands and/or regulating the system. The Regulate System and Protect Equipment function analyzes the inputs and state to determine the changes necessary to bring the controlled system into conformity. The Issue Control Commands function sends signal to the controlled system.

\subsection{Traditional Control System Functional Flow}

Most traditional control systems follow a similar flow to accomplish the required functions. One method for depicting this traditional flow is shown in Figure 3. The controlled system provides monitored system inputs to be managed and processed. The system response and system state are also managed and processed to provide operator feedback, provide information to external networks, and regulate system or issue control commands.

\subsection{Traditional Control System Operational Flow and Key Features}

Traditional controls may also be thought of in terms of data collection and controls, data processing, and human systems interfacing as shown in Figure 4. Data collection and controls monitor the system status and process sensors. Data processing is very rudimentary in traditional controls and allows for single and limited multiloop regulatory control. Logic is available for interlocking with alarms settings. The information received from the system status and process sensors is analyzed and provided to a user through a common interface. The user is not usually targeted with the information, but is presented with a large volume of data to sift through. The user may be an operator, engineer, manager, or other person of a need to know status. Through the common interface, the human provides input to the control system to help regulate and provide equipment protection. Control devices implement the updates through redundancy, fault mitigation and perhaps other processes. Cyber security, nonproliferation sensors, and physical security sensors are in place as bolt-on and are not integrated into the control system design as a holistic process.

A key feature of traditional control systems is the integration of operations. The operator of a traditional control system can view necessary information all from a single terminal. Another feature is large amounts of data exists to provide basis for analysis of systems implementing traditional controls and many operators have been trained to manage this type of controls. 


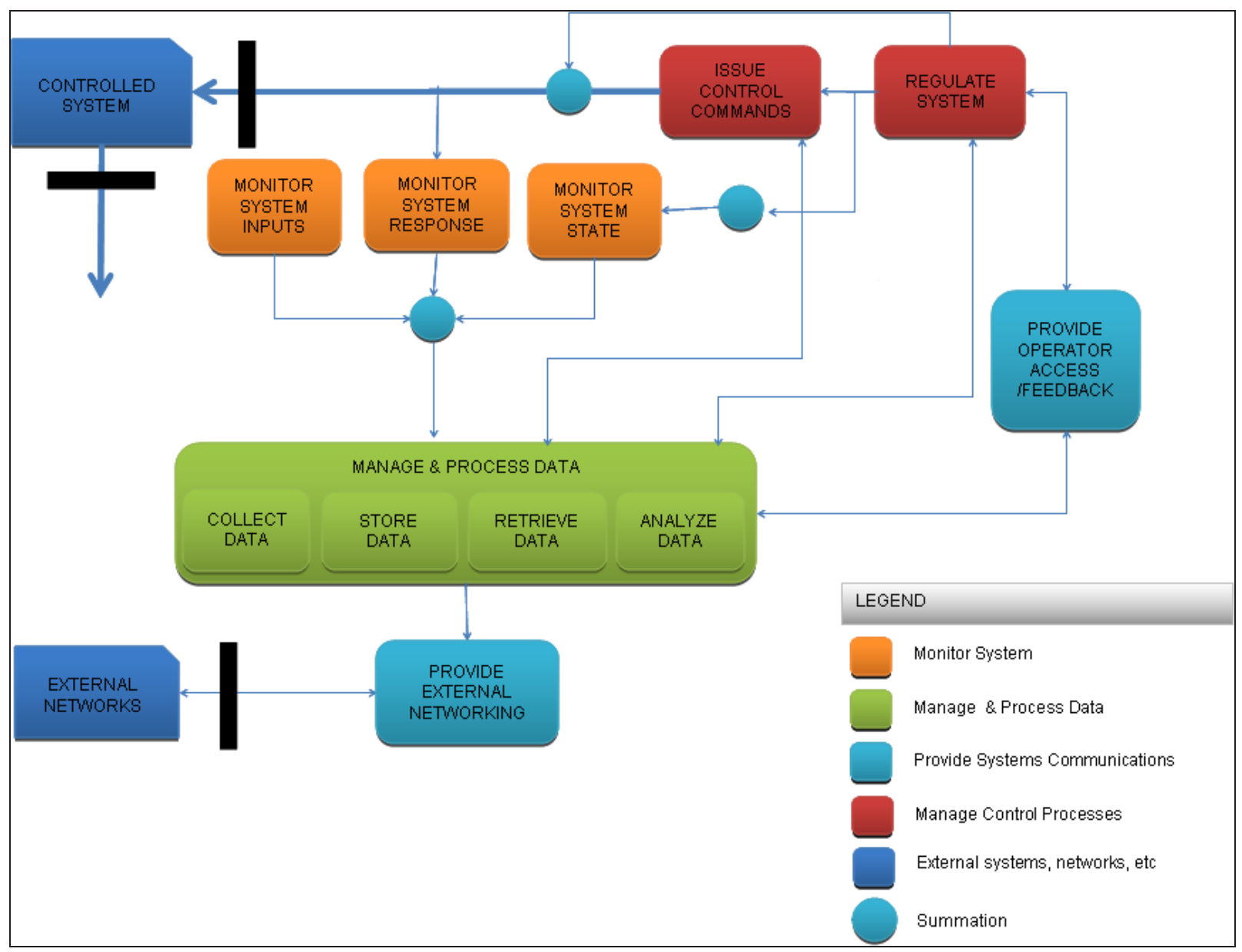

Figure 3. Traditional controls functional flow.

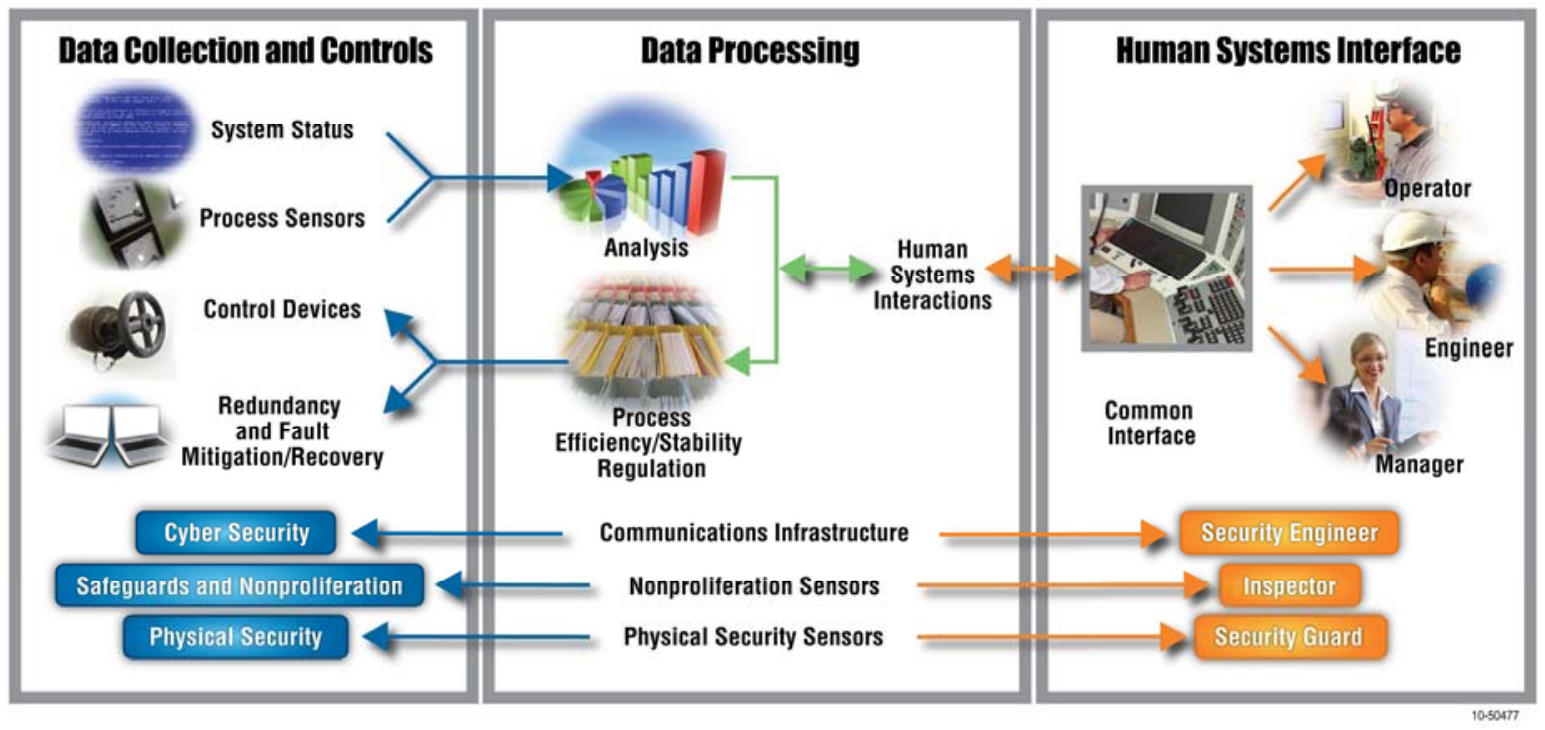

Figure 4. Traditional control system operational flow. 


\section{RESILIENT CONTROL SYSTEMS}

Resilient design necessitates a paradigm shift with respect to the methods historically used in control system design. The move from reactive to proactive control of plants and mechanisms, by which the evaluation and verification of designs is considered all the way from design through implementation stages of resilient control systems, is enabled by this paradigm shift. This section contains a high-level description of resilient control systems implementation along with common resilience terminology.

\subsection{Resilient Control System Functional Framework and Definitions}

A resilient control system may be defined as a controls system which considers the multiple facets of requirements that drive the performance of control systems in a holistic fashion, whether they are security or stability, stability or efficiency, human interactions or complex interdependencies. A resilient control system may also be defined as a controls system "that maintains state awareness and an accepted level of operational normalcy in response to disturbances, including threats of an unexpected and malicious nature," 4 as shown in Figure 5.

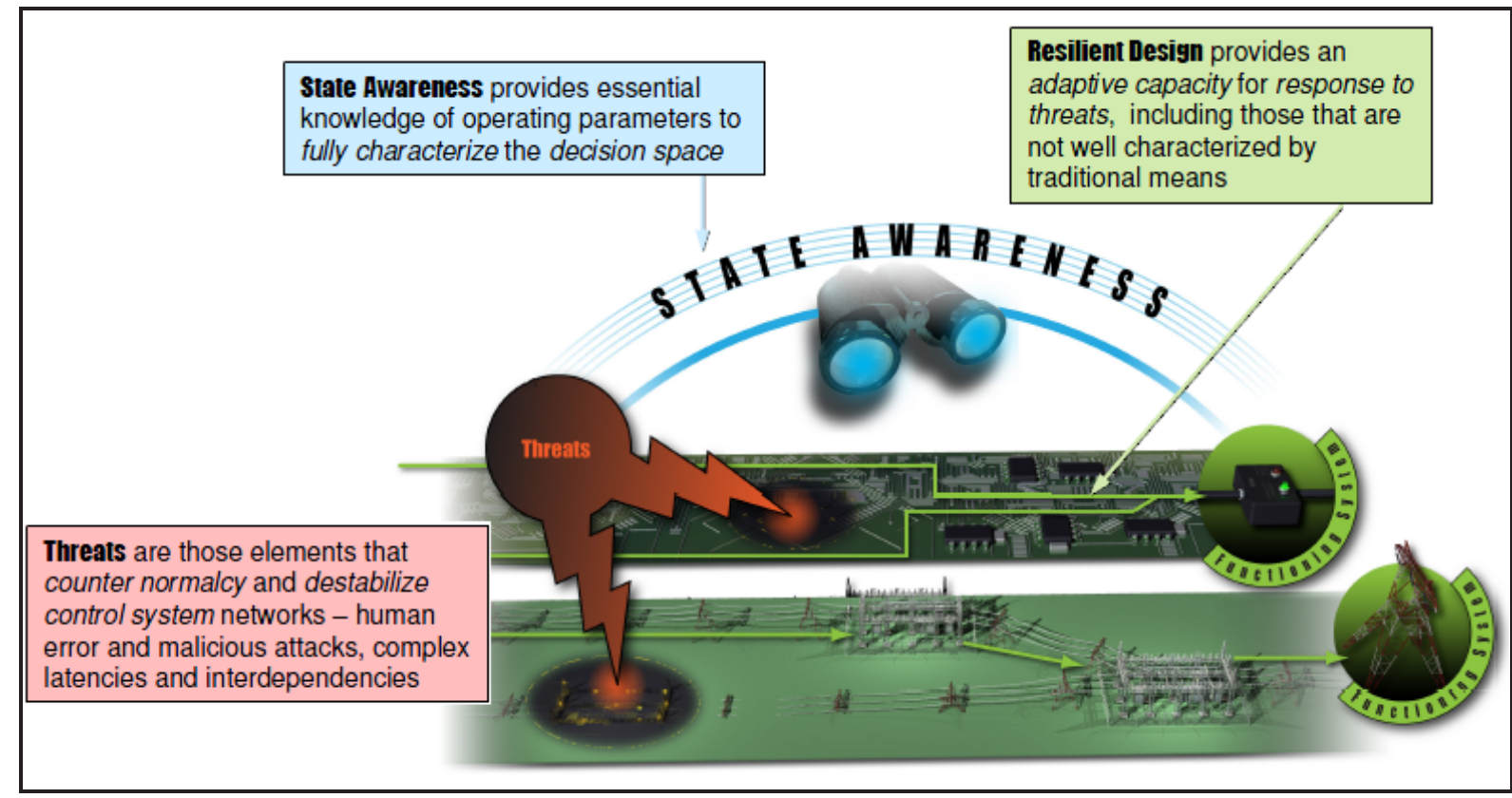

Figure 5. State awareness, resilient design, and threats.

A resilient control system has a similar functional framework to the traditional controls framework as shown in Section 2. The differences between the two exist in the depth, capabilities, and philosophical goals of the two types of controls such as the holistic integration of performance parameters in resilient controls versus the separate, global situational awareness in traditional control systems. Another difference is the consideration of human benefits in resilient control systems versus seeing human error in traditional control systems. These differences are discussed in this section and the practical ramifications of these differences are discussed in detail in Section 5.

A resilient control system monitors the system, manages and processes data, provides system communications, and manages the control processes in a secure, proactive, and adaptive way. Fundamental monitoring and traditional control principles applied to achieve a level of success in preventing security events are primarily reactive. A goal achieved by resilient controls is to be proactive not reactive in controlling plants. The basis of resilient design requires consideration of all threats, all 
steps required to determine proper operation, and counteractive measures to assure proper operation. These measures, categorized as cyber and physical security, process efficiency and stability, and process compliancy, provide the operating requirements monitored for state awareness. ${ }^{2}$

Resilient controls consider the multiple facets of requirements that drive the performance of control systems in a holistic fashion whether they are security or stability, stability or efficiency, human interactions, or complex interdependencies. A resilient control system considers a wide range of requirements to develop an adaptive capacity to complex events that can lead to failure of traditional control system designs as shown in Figure 6.

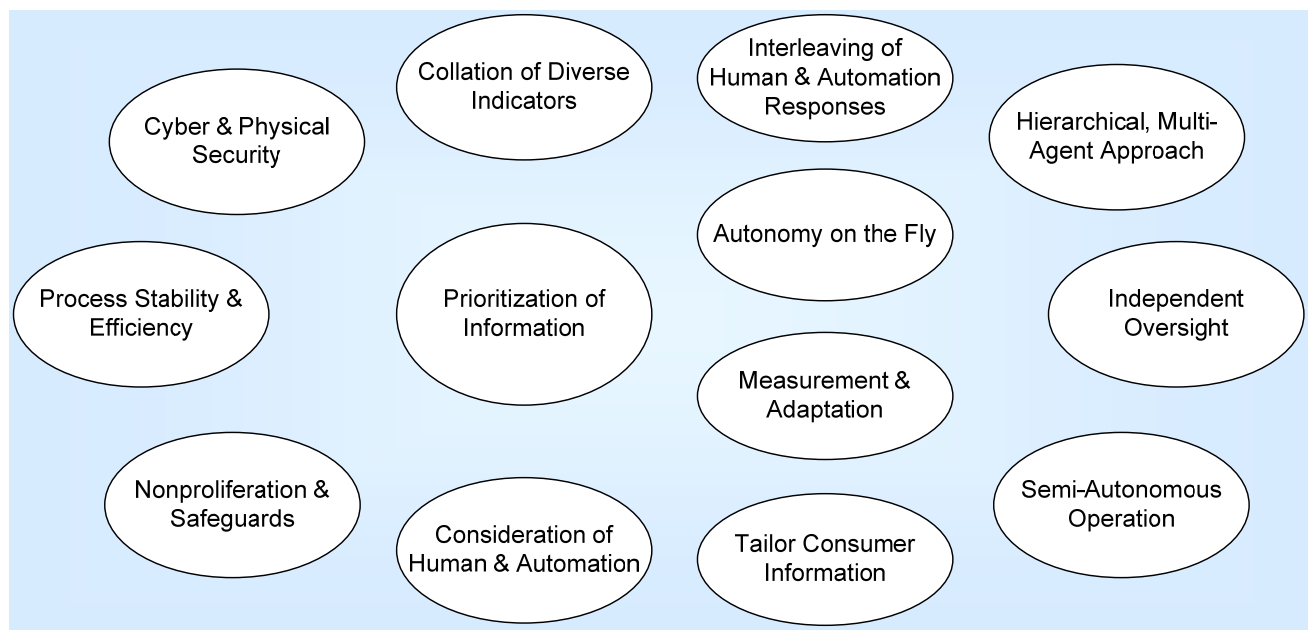

Figure 6. Additional functions performed by resilient controls.

In addition to the capabilities of traditional control systems discussed in Section 2, a resilient control system framework encompasses two areas: state awareness and resilient design as illustrated in Figure 7. State awareness provides the plant operator with a means of understanding the resilience and stability of systems in real time and the extent to which the operator can rely upon the presented information. Areas of resilience include cyber awareness, human systems, and complex, networked control systems. A notional measure of resilience is in the adaptive capacity or the ability to respond to a threat, remain resilient, and maintain minimum acceptable functionality.

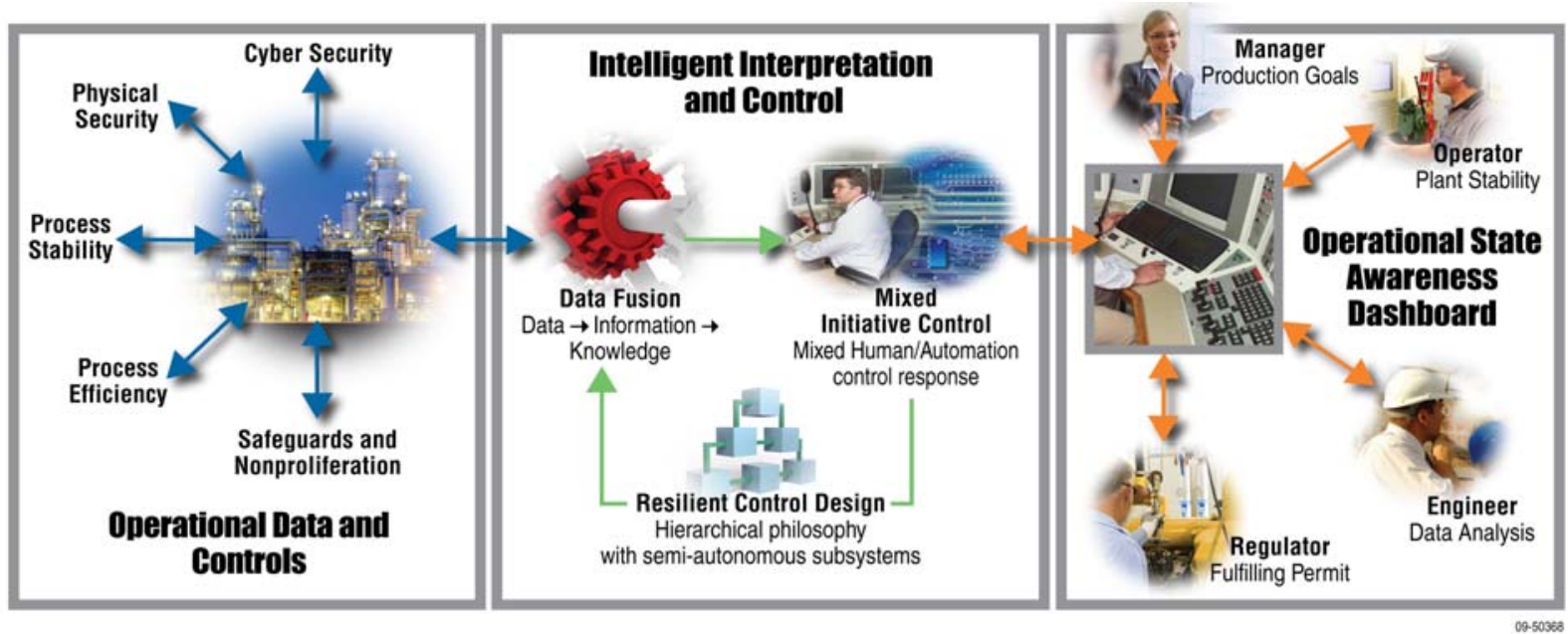

Figure 7. Resilient control system. ${ }^{6}$ 


\subsection{Resilient Control System Functional Flow}

One method for depicting the traditional functional flow is shown in Section 2.2. A resilient control system usually follows this traditional functional flow while implementing the key features and functions of resilient controls in additional to the functions of traditional controls. Similar to those in traditional control systems, resilient control system inputs are managed and processed and the system's response and state are managed and processed to provide operator feedback and information to external networks and to regulate the system or issue control commands.

In addition to the traditional monitor system functions and processes for collecting state, input, and response data to measure a controlled system, a resilient control system also monitors and anticipates internal system (such as instrument readings and states of the control system), and global functions of the system. A resilient control system predicts and anticipates abnormal behavior resulting in a proactive design. In resilient controls, the subsystems are globally optimized (autonomy configuration) and future optimization is anticipated resulting in a fluid system that is able to optimize while running. Resilient control systems are capable of predicting and locating the cause of system failures before the fact and then provide signals inferred from system data to relevant entities verses providing only the received signals in traditional controls systems. Resilient controls systems provide adaptive capacity for degraded signals (Improve Signal Quality) based on information quality judgment, thus overcoming brittleness arising from sensor dependencies.

Unlike traditional control systems, resilient control systems will be designed, installed, operated, and maintained to survive a natural disaster, human error, or intentional cyber attack with no loss of critical function. This is no small challenge in a sector that is complex, highly networked, and sensitive to the mildest failure. Research is required to achieve resilience, address the threats in next generation control systems, and integrate an understanding of cyber security, human interaction, and complex network design. The integration of these three aspects will be introduced in the concepts of data fusion, mixed initiative, and hierarchical control system design.

Data fusion plays a significant role in tailoring information to the user and provides a broader state awareness for items such as malicious cyber attack. Data fusion technology can also integrate diverse forms of data, allowing contrast or variations from normalcy to be recognized. With highly integrated combinations of multiple control systems, data fusion research is needed to quickly recognize human error or malicious cyber attack and prevent a negative impact cascading to multiple process systems. A data fusion approach can process diverse data to proactively recognize threats within each measure of normalcy and prioritize responses.

Mixed initiative control provides an optimized combination of automation and human response to achieve the most resilient reaction from both. Humans can be more effective at recalibrating response to changing environments, but must be effectively modeled to ensure the proper integration with automation. The mixed initiative framework shown in Figure 8 is proposed to provide mechanisms to integrate automation and human response in an optimized manner, taking benefit from the inherent resilience in both.

When considering the integrated operation of varied processes with competing objectives, a hierarchical approach to control system design lends resilience. A hierarchical, multiagent approach ensures the required overall performance is maintained according to the measures by which normalcy is accessed (as it considers the integrated operation holistically). A hierarchical, multiagent approach provides a global perspective to complex system design, which can be delegated appropriately to lower echelons along with a predefined level of control autonomy. A hierarchical, multiagent control system design provides a nonfragile mechanism for optimizing control system performance. 


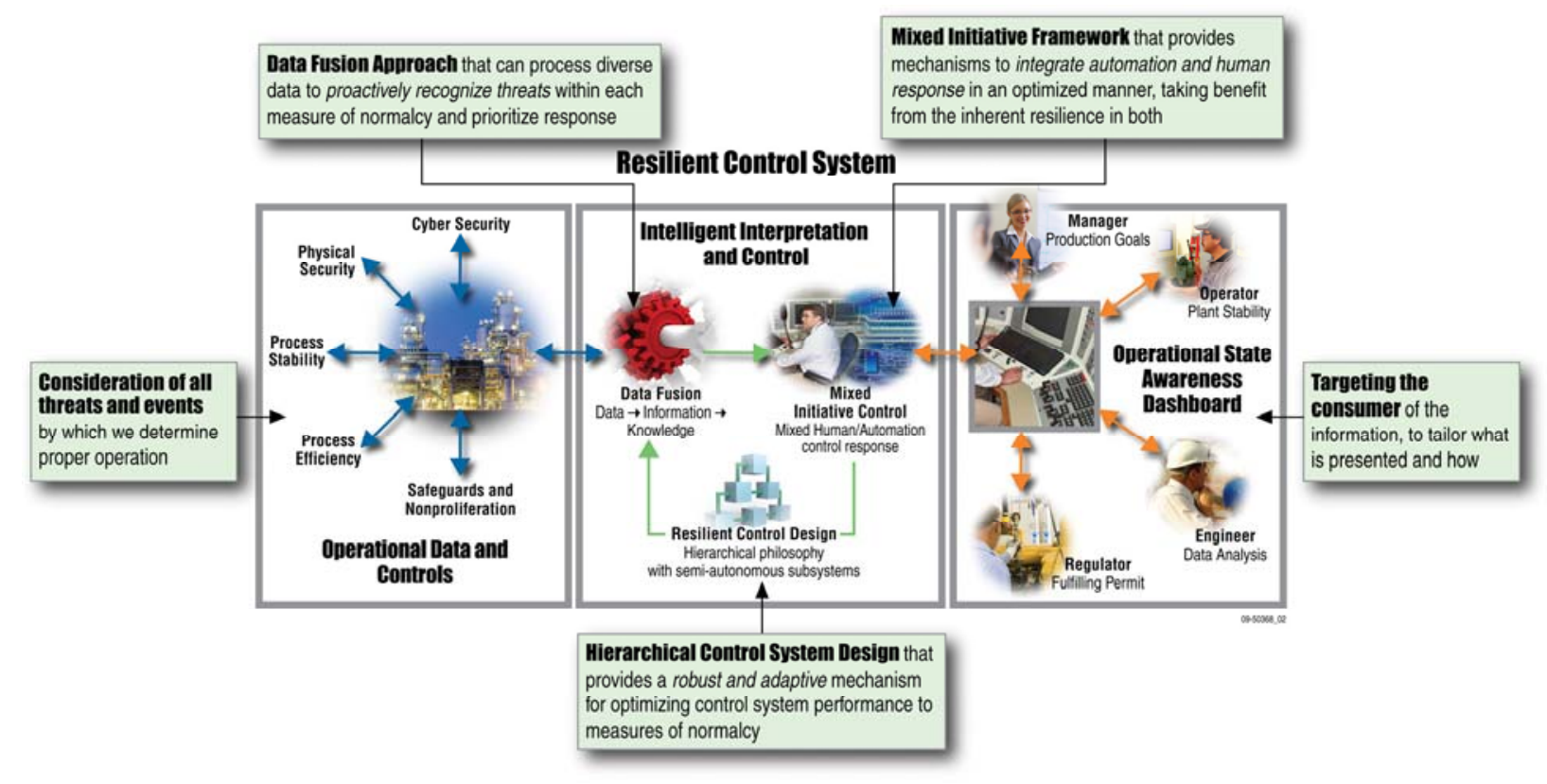

Figure 8. Resilient control system framework.

In addition to the traditional system functions and processes used to manage and process data, resilient control systems collate diverse indicators to increase data confidence. A resilient control system updates and refines the future system environment model to aide in predicting future events. Resilient controls prioritize information by reducing data to the necessary information, identifying event causes, and improving system characterization as shown in Figure 9.
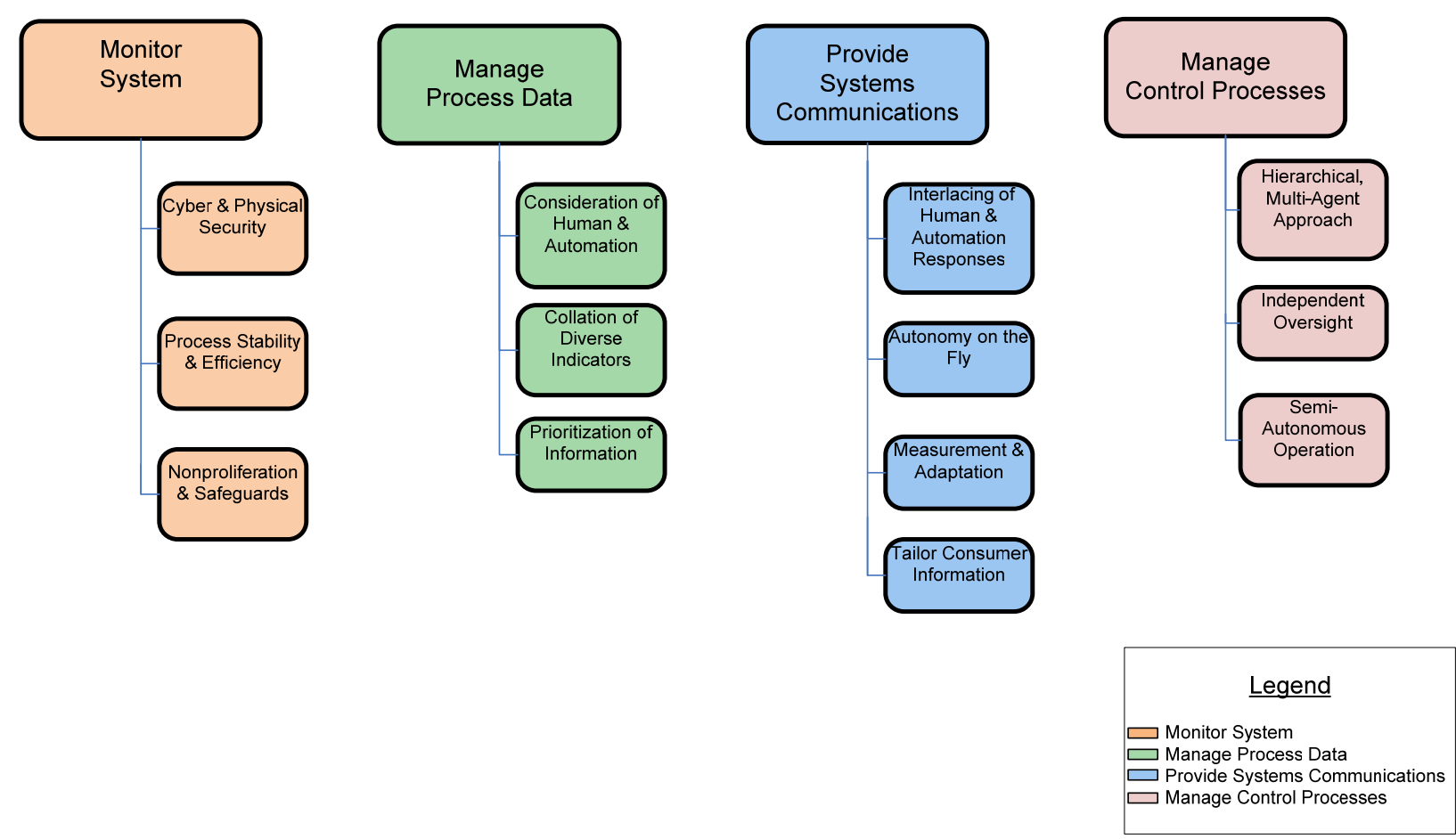

Figure 9. Functional flow additions of resilient control systems. 
In addition to providing the systems communications functions and processes found in traditional controls, resilient controls provide operator access and feedback, which enables communications between the control system and a human operator. Resilient controls provide advanced cyber security integration into multiple levels of data fusion and communication and report to the human systems interface. A resilient control system is able to identify and distinguish between normal and abnormal communications and ensure the integrity of the cyber and physical environment by adaptively detecting threat and authenticating data. Resilient controls provide layers of detection and network security responses to refine and prioritize discrete targets of interest. Resilient controls provide randomized system attributes to confound attacks while maintaining determinism for the control application and provide user adapted information access and feedback. Joint human and automation cognitive decision making is also facilitated by resilient controls for optimal reaction.

In addition to managing control process functions performed by traditional controls, resilient controls distribute system resources and execute internal operations in real time to allow users to locate and maintain awareness of materials and resources. Resilient controls proactively model the future control system and its environment. Human system interactions of resilient controls interlace human and automation responses for mixed initiative control. Mixed initiative controls provide a customized interface screen for users and mixes human with automation for optimal reaction, including the use of human sensors and correlation. Resilient controls validate and prioritize task execution and regulation responses based on the load and history in a changing environment. Resilient controls provide user measurement and adaptation data in the form of inferred signals so the user has greater insight into the current system and the future system. A resilient system has signals that are collated, predicted, and inferred to globally optimize the system and provide a future and predictive controls environment.

\subsection{Resilient Control System Operational Flow and Key Features}

While a resilient control system follows a similar operational flow to a traditional control system, there are many differences in how threats are addressed, the state awareness level, and the implementation level of resilient design. These differences provide a significant delta in the operational normalcy of the system. As shown in Figure 10, the additions of cyber security, safeguards and nonproliferation, and increased physical security overarch the functions of the system.

Key features of resilient controls systems include increasing the state awareness and moving from reliable to resilient design. A resilient control system considers all threats and events by which proper operation is determined. Increased state awareness is achieved in a resilient control system by many methods, including the following:

- Cyber and physical security:

- Cyber awareness requires that the control system report when a systems has been compromised

- Data authentication and diversity to ensure integrity of cyber and physical environment

- Layers of detection fidelity to refine and prioritize discrete targets of interest

- Development of analytics that identify normal communications behavior for wired

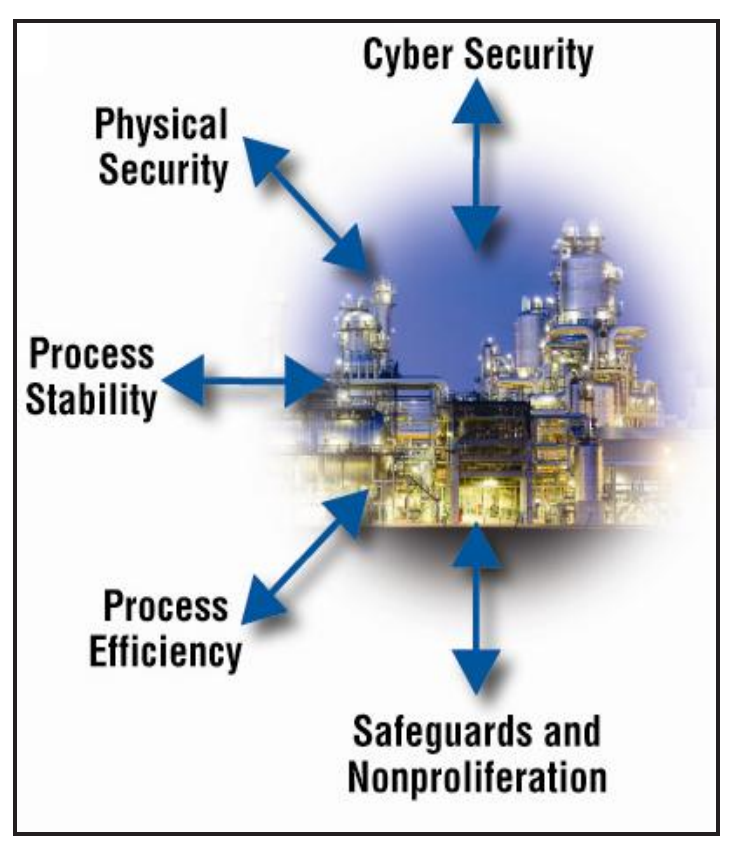

Figure 10. Key features of resilient control systems. 
and wireless

- Randomization of system attributes to confound attacks while maintaining determinism for the control application.

- Process stability and efficiency:

- Predict and diagnose deviations from nominal behavior, migrating from reactive to proactive design

- Optimize subsystems to a global maxima or minima, providing a holistic perspective in ensuring plant safety and efficiency

- Build upon supervisory control, which considers the dynamic interactions of plant systems and incorporates advanced control theory

- Provide adaptive capacity based on an information quality judgment, overcoming brittleness arising from sensor dependencies

- Nonproliferation and safeguards:

- Timely knowledge of the location and movement of nuclear material within a nuclear facility

- Real-time knowledge of plant activity to prevent downtime of facilities, and diversion or misuse of nuclear material

- Process accountancy to provide enhanced awareness of the nuclear material inventory and status

Resilient design, a key feature of a resilient control system, goes beyond being just reliable by many techniques including data fusion, mixed initiative, control system, state dashboard, and human system. Resilient controls has the ability to anticipate as well and predict and diagnose abnormal behavior. Data fusion aides a resilient design by numerous methods, including the following:

- Consideration of human and automation to determine algorithms and methodologies

- Collation of diverse indicators, such as cyber security and process data, performed to determine desired operation

- Proper prioritization of the information provided to the consumer to aid recognition and action

- Reduction - the reduction of data to provide only that information necessary to achieve the appropriate response

- Identification - validation and invalidation of causes for events, e.g., a process upset caused by a failed valve and not cyber attack

- Improved characterization and knowledge - development of new information that helps to better characterize state.

Mixed initiative aides a resilient design by numerous methods, including the following:

- Interlacing human and automation responses for optimal reaction

- A control system concept that allows the user to configure autonomy on the fly

- Measurement and adaptation to the user based on current understanding, performance, and changing environment

- Provides for prioritization of tasks based on time load and historic resilience.

- The control system aides a resilient design by numerous methods, including the following:

- Hierarchical, multiagent approach to supervisory design that considers the control system and affected operation holistically 
- Independent oversight outside of echelon insures state awareness is accurately communicated up and philosophy is communicated down

- Semiautonomous operation allowed within defined boundaries for lower echelons

- Traditional concepts are helpful, including redundancy and diversity, but must be extended to consider all threats.

State Dashboard aides a resilient design by numerous methods, including the following:

- Interactions between humans and the interface form the basis for proper or improper judgments

- Authorizing the interaction level by individual certifications and responsibilities, and measured performance

- Presentation information must reflect the needs of the consumer, whether operator, manager, or engineer, and their responsibilities.

- Human systems aides a resilient design by numerous methods, including the following:

- The human psyche can have the ability to quickly understand novel situations, with the potential of providing additional control system resilience

- Human behavior is not reproducibly predictable; unique measurement and correlations of performance are required

- Bayesian methods provide a way to take into account evidence regarding human response, but is one of many approaches

- Defining methods of mixed initiative response, a combination of human and automation, can provide more resilience during complex operations. 


\section{HTGRS APPLICABLE OPPORTUNITIES}

Traditional reactor control and safety related reactor protection systems have extensive and thoroughly vetted requirements. Implementing a resilient strategy above and beyond the existing traditional reactor control and safety related rector protection systems does not negate or challenge any of those existing requirements. A resilient strategy seeks to improve availability and enhance the economic competitiveness of the reactor.

This section identifies existing gaps between a traditional control system and a resilient control system as applied to HTGRs, and presents several of the many benefits resilient controls will have on HTGRs.

To identify the gaps, traditional control systems and resilient control systems both underwent functional analysis. This analysis highlighted differences in the measures of normalcy, data fusion, resilient control design, mixed initiative control, and adaptive capacity for each item in the controls functional framework. The gap analysis process is shown in Figure 11.

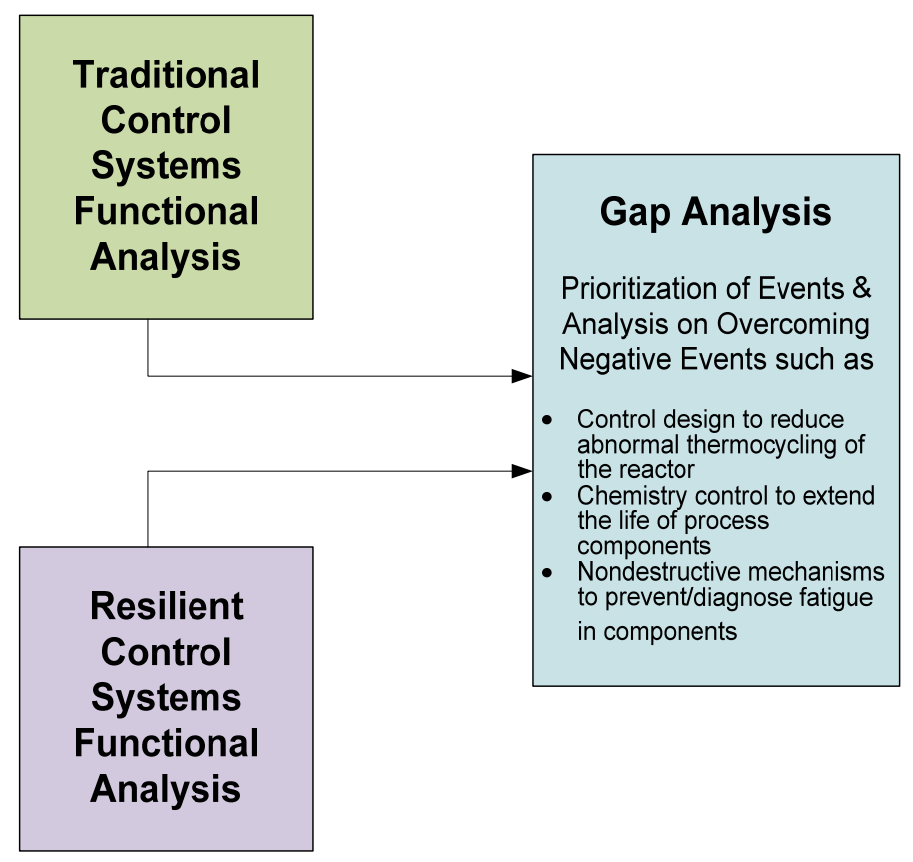

Figure 11. Gap analysis process.

\subsection{Monitor System Gap Analysis}

The additional functions required to move from a traditional to a resilient control system in performing the monitor system function are numerous. The functions performed by a traditional control system are shown in on the left side of Figure 12, shown in dark orange. A resilient controls system performs these functions in addition to the functions on the right side of Figure 12, shown in light orange. 


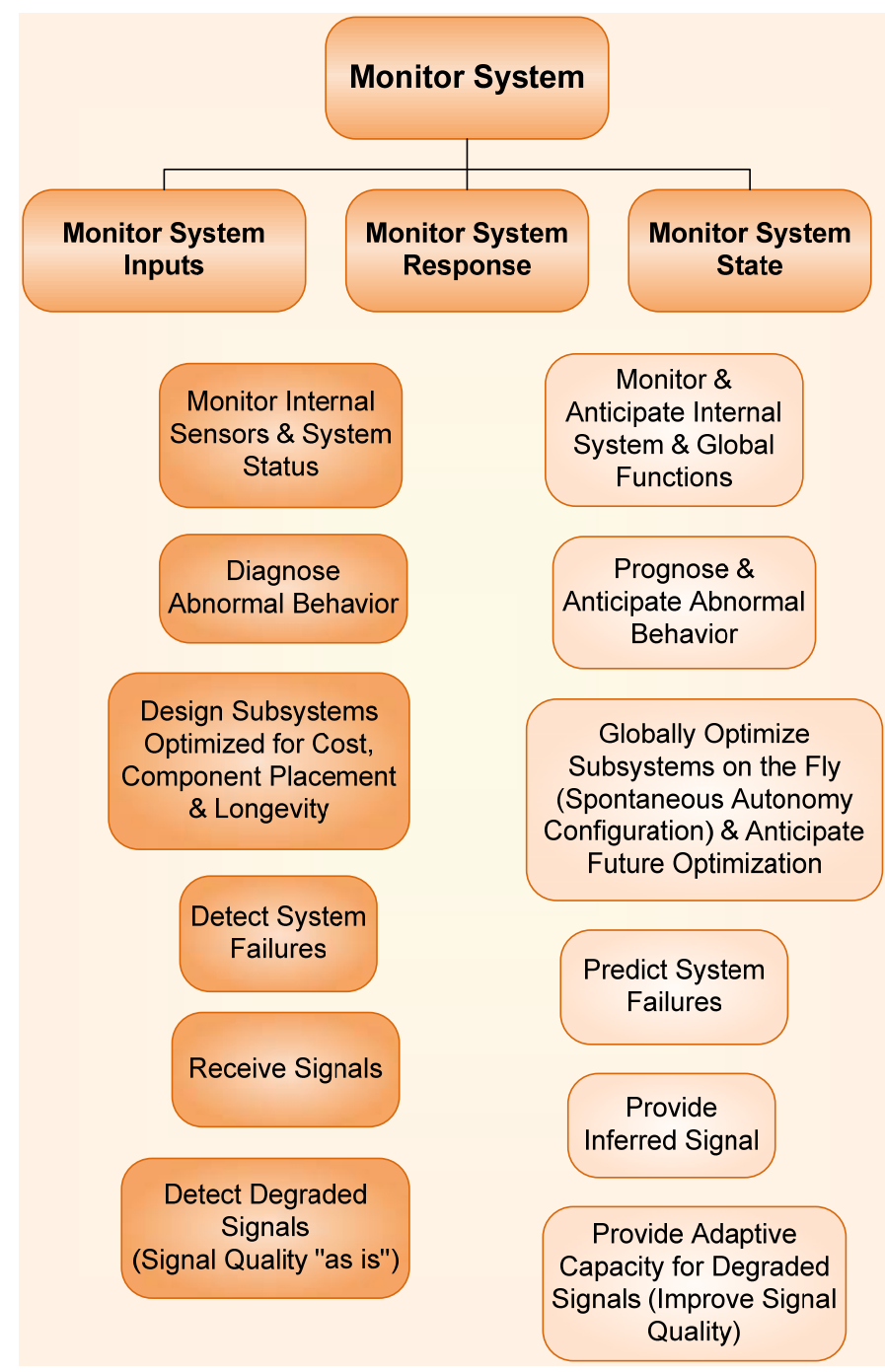

Figure 12. Monitor system gaps.

A resilient control system also monitors and anticipates internal system (such as instrument readings and states of the control system), and global functions of the system. Traditional controls diagnose and perceive abnormal behavior by detecting and locating the cause of past abnormal signals resulting in a reactive design. Resilient controls predict and anticipate abnormal behavior resulting in a proactive and predictive design. Resilient controls employ online condition monitoring, which looks at data and diagnoses abnormal behavior through a multitude of different analysis techniques. Once the behavior has been characterized, resilient controls predict failure, maintenance needs, or control to prevent behaviors. Traditional controls systems are usually design subsystems optimized for cost, component placement, and longevity. In traditional controls, fixed optimization is created during the design phase. In resilient controls, the subsystems are globally optimized on the fly (autonomy configuration) and future optimization is anticipated resulting in a fluid system able to optimize while running.

Traditional controls detect and locate the cause of system failures after the fact. Resilient control systems are capable of predicting and locating the cause of system failures before the fact. Resilient controls systems also provide signals inferred from system data and provided to relevant entities verses providing only the received signals in traditional controls systems. 
Traditional control systems detect degraded signals (Signal Quality "as is") but are unable to improve them. Resilient controls systems provide adaptive capacity for degraded signals (Improve Signal Quality) based on information quality judgment, overcoming brittleness arising from sensor dependencies.

When monitoring the system, resilient controls consider all threats and events by which proper operation is determined. Features not included in traditional controls systems include cyber and physical security, process stability and efficiency, and nonproliferation and safeguards as shown in Figure 13.

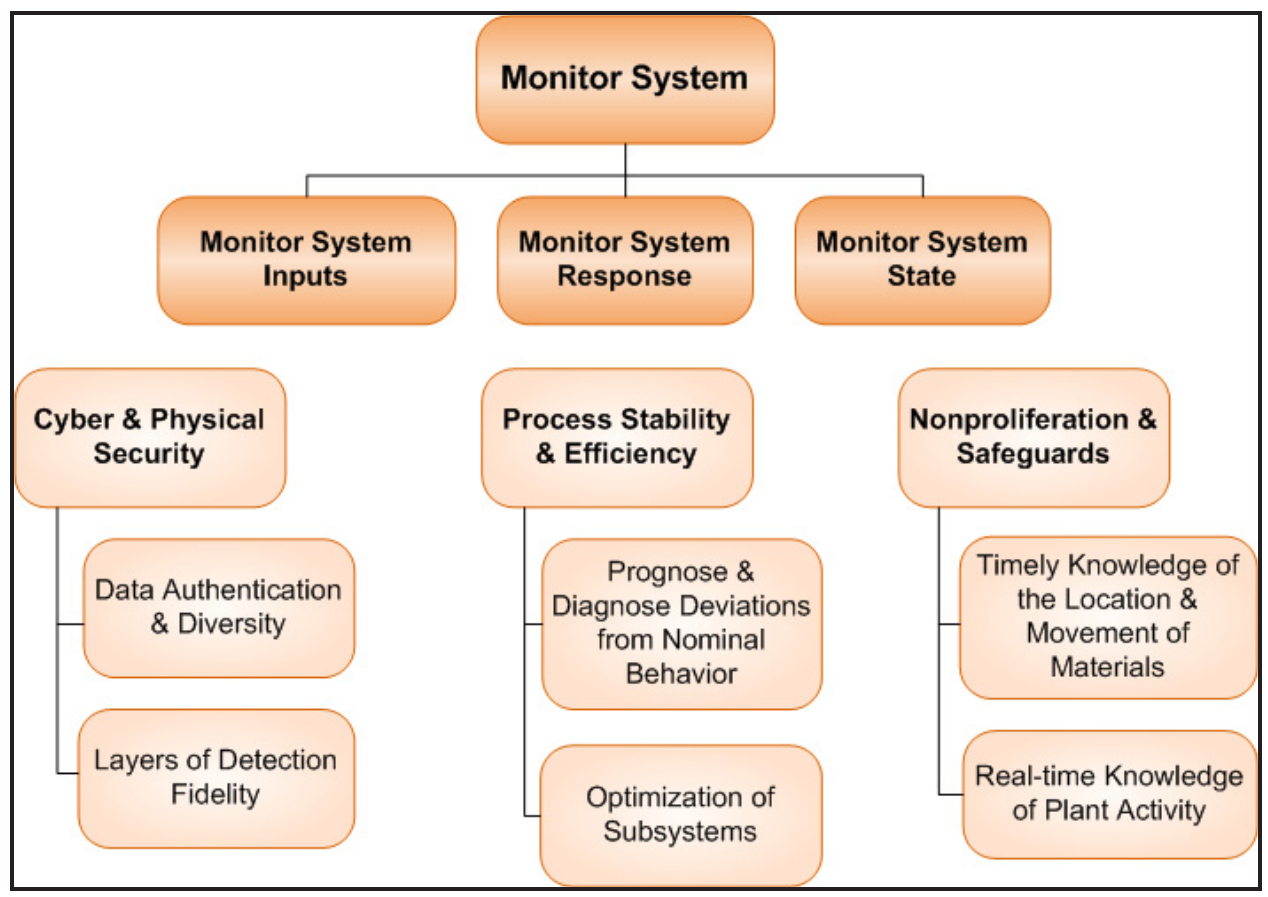

Figure 13. Monitor System.

Cyber and physical security provide data authentication and diversity to ensure the integrity of cyber and physical environments. Cyber and physical security also employ layers of detection fidelity to refine and prioritize discrete targets of interest. Process stability and efficiency predict and diagnose deviations from nominal behavior, migrating the design from reactive to proactive. The optimization of subsystems to a global maxima or minima, providing a holistic perspective in ensuring plant safety and efficiency, is also a feature of process stability and efficiency. Nonproliferation and safeguards provide timely knowledge of the location and movement of nuclear material within a nuclear facility. Real-time knowledge of plant activity prevents downtime of facilities and the diversion or misuse of nuclear material.

\subsection{Manage and Process Data Gap Analysis}

The additional functions required to move from a traditional to a resilient control system in performing the Manage and Process Data function in traditional and resilient control systems are numerous. The functions performed by a traditional control system are on the left side in Figure 13, shown in dark green. A resilient control system performs these functions in addition to the functions on the right side of Figure 14, shown in light green. 


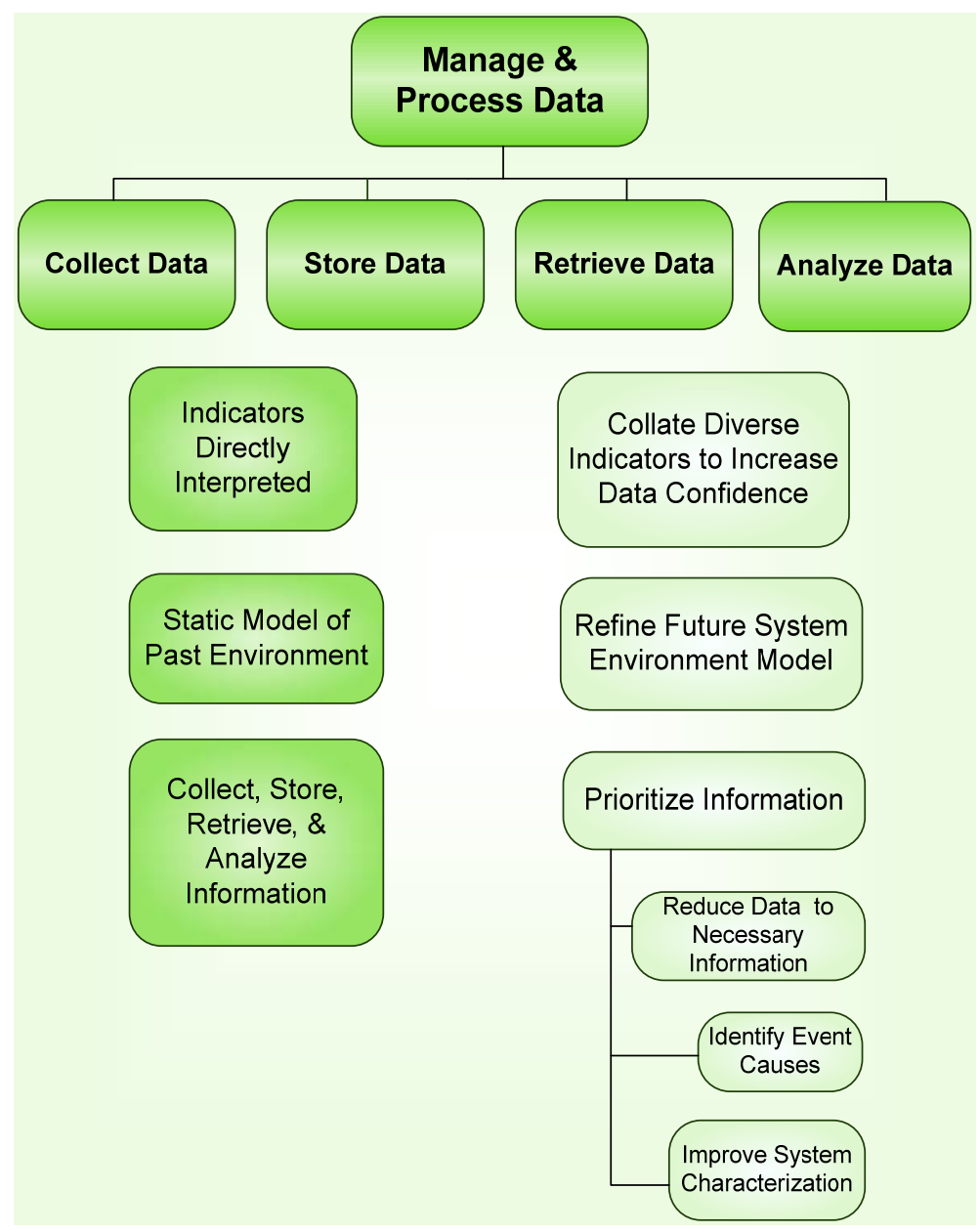

Figure 14. Manage and process data gaps.

A traditional control system directly receives and interprets indicator signals from raw analog or digital transmissions from instruments or observational sensors. Resilient control systems collate diverse indicators to increase data confidence. A traditional control system operates from a fixed, static model of the system created in the past. A resilient control system updates and refines the future system environment model to aid in predicting future events. In addition to collecting, storing, retrieving, and analyzing information the way a traditional control systems does, a resilient control system prioritizes information by reducing the data to the necessary information, identifying event causes, and improving system characterization.

When managing and processing data, resilient controls can fuse diverse process data to proactively recognize threats within each measure of normalcy and prioritize response as shown in Figure 15. Resilient controls considers both human input and automation to determine algorithms and methodologies. Resilient controls collate diverse indicators, such as cyber security and process data, to determine the desired operation. Resilient controls also priorities the information provided to the consumer to aid recognition and action. 


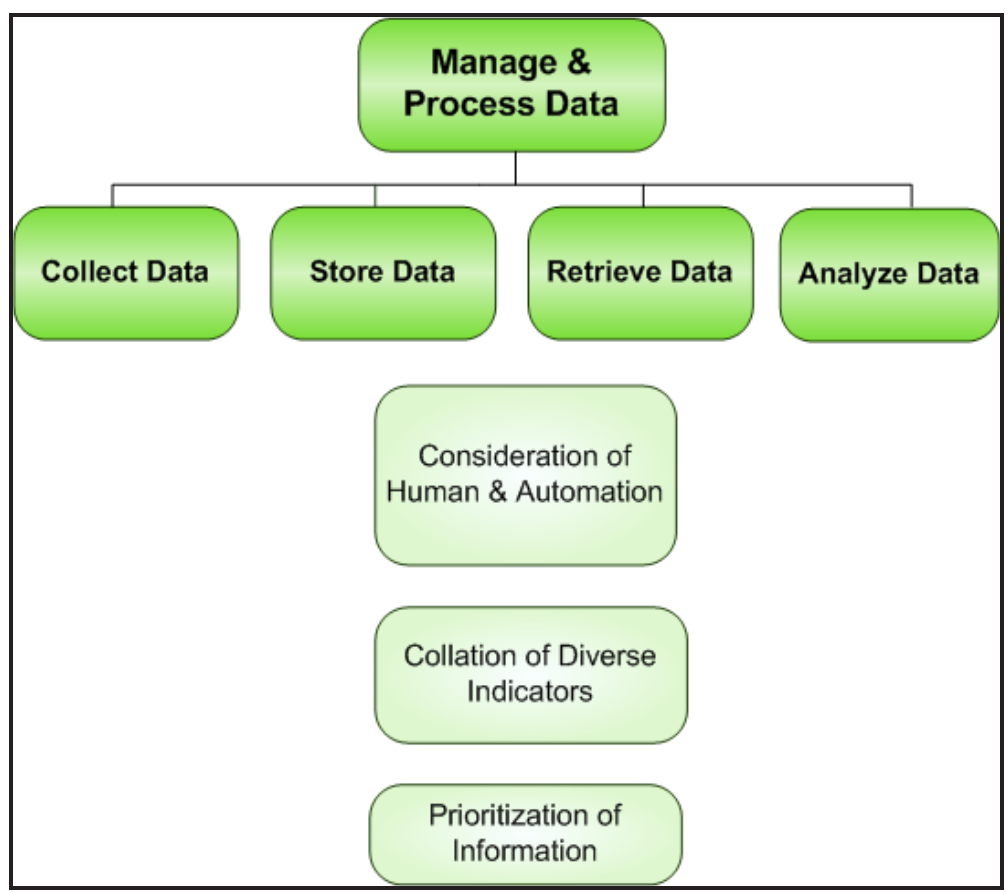

Figure 15. Manage and process data.

\subsection{Provide Systems Communication Gap Analysis}

The additional functions required to move from a traditional to a resilient control system in performing the Provide Systems Communications function in traditional and resilient control systems are abundant. The functions performed by a traditional control system are on the left side of Figure 1, shown in dark blue. A resilient controls system performs these functions in addition to the functions on the right side of Figure 14, shown in light blue.

A traditional controls system provides cyber security for data collection and controls and reports these findings to a security engineer. A resilient control system provides cyber security for data collection and controls and advanced cyber security integration into multiple levels of data fusion and communications and reporting to the human systems interface. In a traditional control system, communications continue as is and are assumed normal. A resilient controls system is able to identify and distinguish between normal and abnormal communications. A traditional control system assumes data is authentic and responds to threats after they are identified. In a resilient control system, threats are adaptively detected and data is authenticated to ensure cyber and physical environments are safe and secure. A traditional control system has preset responses to a preset list of threats, whereas a resilient control system has layers of detection fidelity to refine and prioritize discrete targets of interest. A traditional control system has preset and unchanging system parameters, whereas a resilient control system has randomized system attributes to confound attacks while maintaining determinism for the control application. A tradition controls system has a universal maintenance screen interface, which utilizes the same interface screen for any user.

A resilient control system provides user adapted information access and feedback, which utilizes a user interface screen and information adapted to the individual user. In a traditional control system, either the operator or automatic response system makes the decision, not both. A resilient system facilitates joint human and automation cognitive decision making for optimal reaction. 


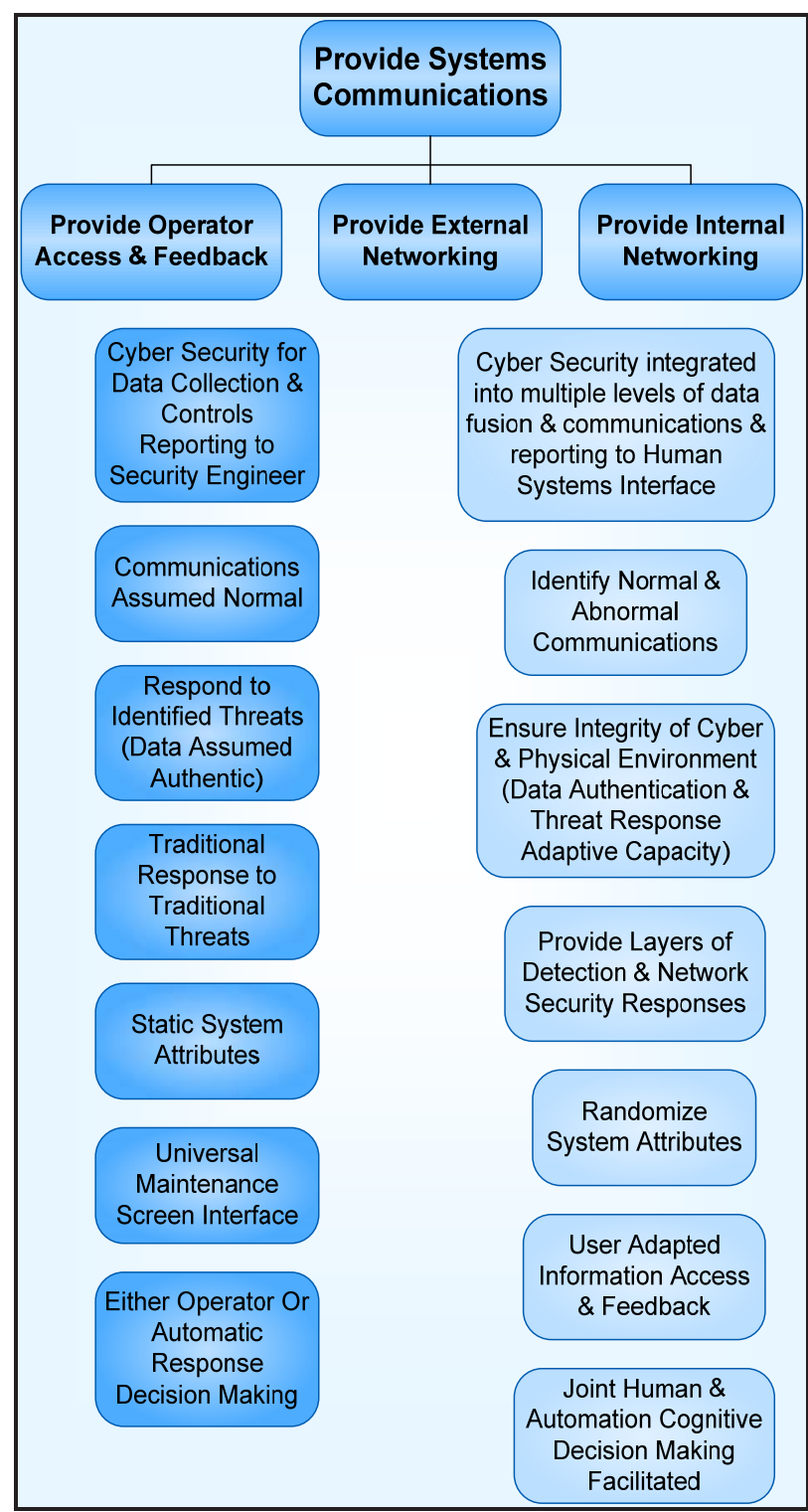

Figure 16. Provide Systems Communications Gaps.

As shown in Figure 17, when providing systems communications, mechanisms in resilient controls integrate automation and human response in an optimized manner, benefiting from the inherent resilience in both by interlacing of human and automation responses for optimal reaction, allowing the user to configure autonomy on the fly and measuring and adapting to the user based on current understanding, performance, and the changing environment. Targeting the consumer of the information, resilience controls tailor what is presented and how interactions between the human and the interface form the basis for proper or improper judgments. A resilient control system authorizes interaction level by individual certifications, responsibilities, and measured performance. Resilient controls present the information in reflection to the needs of the consumer, whether operator, manager or engineer, and his or her responsibilities. 


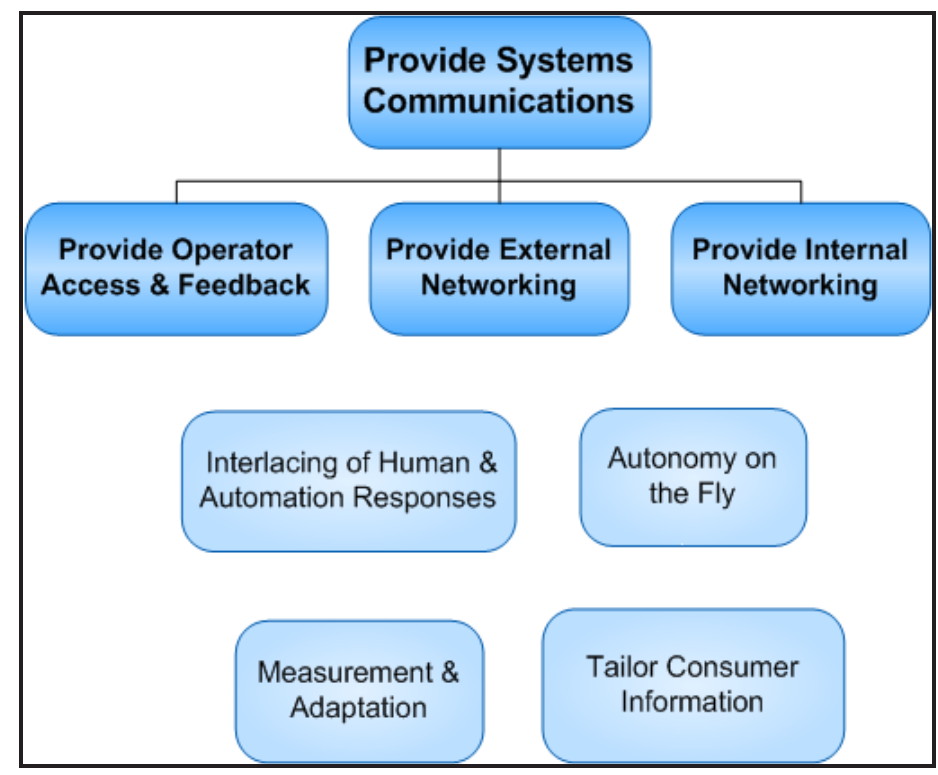

Figure 17. Provide Systems Communications.

\subsection{Manage Control Processes Gap Analysis}

The additional functions required to move from a traditional to a resilient control system in performing the Manage Control Processes function in traditional and resilient control systems are numerous. The functions performed by a traditional control system are on the left side of Figure 18, shown in dark red. A resilient controls system performs these functions in addition to the functions on the right side of Figure 18, shown in light red.

A traditional control system distributes system resources and executes internal operations in a time frame preset as optimal. A resilient control system distributes system resources and executes internal operations in real time, which allows the user to locate and maintain awareness of materials and resources. In a traditional control system, a reactive model of the current control system and environment may exist, whereas a resilient control system proactively and continuously models the future control system and its environment. A traditional control system employs a universal human interface, which is the same for all users and the human system interactions are the common human interface with no human and automation interaction. Human system interactions of resilient control system interlace human and automation responses for mixed initiative control. Mixed initiative control provides a customized interface screen for users and is the mixing of human and automation for optimal reaction including the use of human sensors and human correlation. A traditional control system coordinates system functions based on static, preprogrammed information with unchanging functions preset based on assumptions. A resilient control system validates and prioritizes task execution and regulation responses based on the load, history, and current understanding, performance, and changing environment. In a traditional controls system the user receives information after the fact, whereas is a resilient control system the user measurement and adaptation data in the form of inferred signals is provided so the user has greater insight into the current system and the future system. Supervisory control with redundant signals and fault tolerance, which is reactive and after the fact, is utilized in a traditional control system. A resilient system has signals that are collated, predicted, and inferred to globally optimize the system and provide a future and predictive controls environment. 


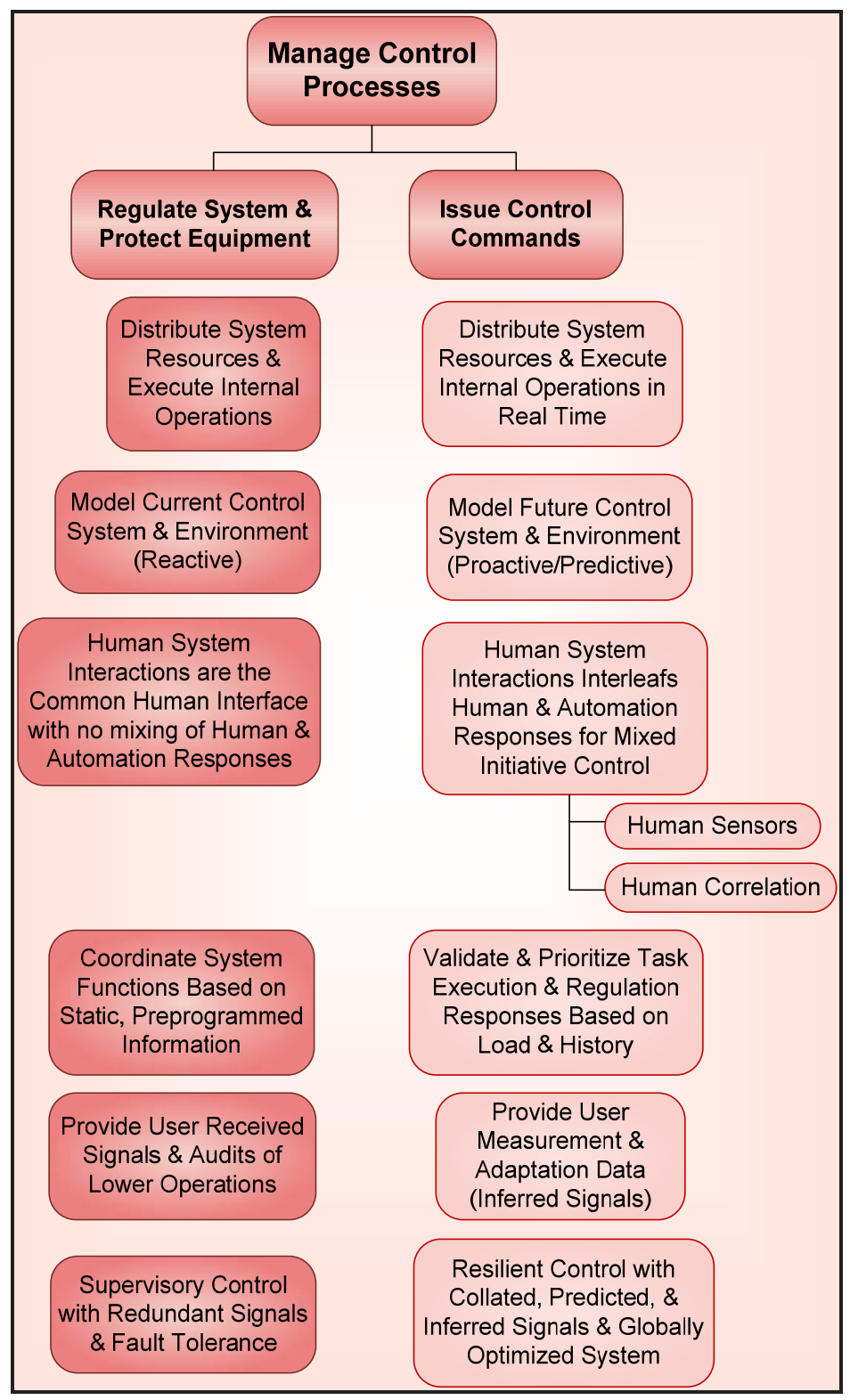

Figure 18. Manage Control Processes Gaps.

When managing control processes, hierarchical control system design provides a robust and adaptive mechanism for optimizing control system performance to measures of normalcy in the following areas as shown in Figure 19:

- Hierarchical, multiagent approach to supervisory design that considers the control system and affected operation holistically

- Independent oversight outside of echelon insures state awareness is accurately communicated up and philosophy is communicated down

- Semiautonomous operation allowed within defined boundaries for lower echelons. 


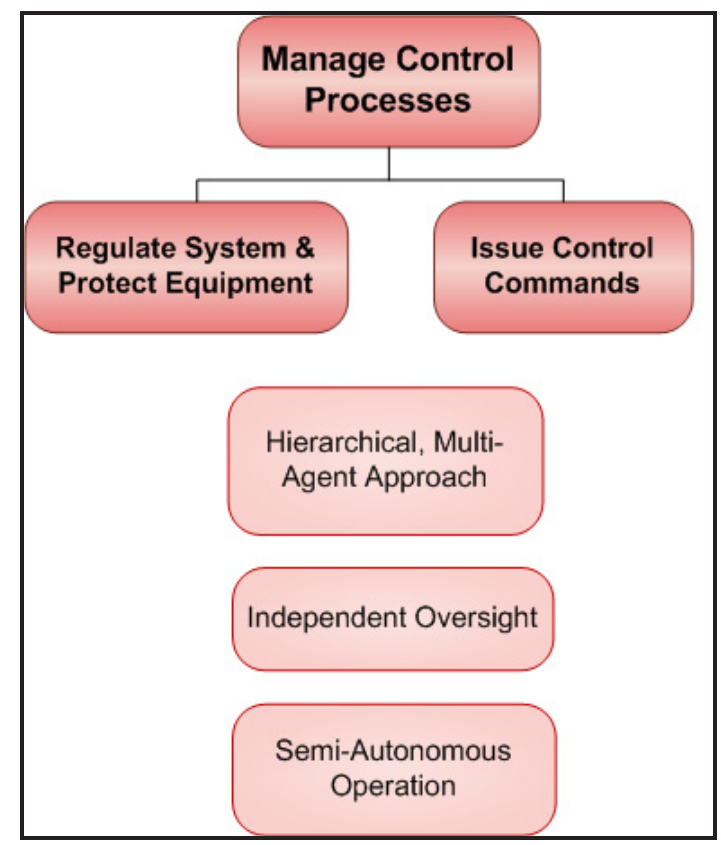

Figure 19. Manage Control Processes.

\subsection{Benefits of Resilient Controls to HTGRs}

Control Systems and their associated instrumentation must meet availability and resiliency parameters. Reliable and resilient control systems will be necessary for the HTGRs to be enhanced economically and provide increased availability.

While fundamental monitoring and control principles can be applied to achieve a level of success in preventing security events, these techniques are primarily reactive. The basis of resilient design requires consideration of all threats and measures by which proper operation is determined. These measures, which can be categorized as cyber and physical security, process efficiency and stability, and process compliancy, provide the operating requirements that are monitored for state awareness.

Resilience considers the multiple facets of requirements that drive the performance of control systems in a holistic fashion, whether they are security or stability, stability or efficiency, human interactions or complex interdependencies. Traditional controls philosophies lack the depth to satisfy these requirements, such as graceful degradation of hierarchical control while under cyber attack or the inferred operating parameters in the event of loss of signal. A resilient control system considers these diverse requirements, thereby developing an adaptive capacity to complex events that can lead to failure of traditional control system designs.

The need for a resilient control system is economic and practical, specifically: extending the longevity of the equipment, making operations more efficient, better utilizing existing operators and support staff, increasing stability when coupling with multiple processes, and integrating process measures such as cyber security and process efficiency. 


\section{REFERENCES}

1. GAO, Critical Infrastructure Protection: Department of Homeland Security Faces Challenges in Fulfilling Cybersecurity Responsibilities, GAO-05-434, May 2005.

2. Craig G. Rieger (Senior Member, IEEE), David I. Gertman, Miles. A. McQueen (Member, IEEE), Notional Examples and Benchmark Aspects of a Resilient Control System, INL/JOU-09-17536, Idaho National Laboratory, Idaho Falls, Idaho, June 2010.

3. “Power," Nuclear News, Vol. 53, No. 4, April 2010, pp. 29.

4. Craig G. Rieger (Idaho National Laboratory), "Control System," Wikipedia, $<$ http://en.wikipedia.org/wiki/Control_system>, Page last modified on 15 July 2010.

5. "Control System," BusinessDictionary.com, $<$ http://www.businessdictionary.com/definition/controlsystem.html>, Copyright(C2010.

6. Craig G. Rieger (Senior Member, IEEE), Notional Benchmark Problems for Resilient Control Systems, INL/JOU-09-17536, Idaho National Laboratory, Idaho Falls, Idaho. 